\title{
INDICATIONS FOR THREE-FIELD DISSECTION FOLLOWED BY ESOPHAGECTOMY FOR ADVANCED CARCINOMA OF THE THORACIC ESOPHAGUS
}

Yoichi Tabira, $\mathrm{MD}^{\mathrm{a}}$

Toshitada Okuma, MD $^{\mathrm{b}}$

Keiichiro Kondo, $\mathrm{MD}^{\mathrm{a}}$

Nobuo Kitamura, MD
Objective: The aim of this study was to evaluate the indication for 3-field lymphadenectomy (3-field dissection) followed by esophagectomy for locally advanced carcinoma of the thoracic esophagus in the presence of lymph node metastasis. Methods: From January 1983 to December 1995, 86 patients with thoracic esophageal carcinoma invading muscularis propria or adventitia underwent radical subtotal esophagectomy after preoperative chemotherapy. Forty-six of the 86 patients underwent a 2 field dissection (mediastinal and abdominal nodes, group A), and 40 patients underwent a 3-field dissection (bilateral cervical, mediastinal, and abdominal nodes, group B). Survival curves were compared between the 2 groups after stratification according to the degree of lymph node involvement (number of positive nodes and involvement of intrathoracic or intrathoracic recurrent nerve chain nodes). Potential prognostic factors of these 86 patients were evaluated by means of Cox regression analysis. Results: There were no significant differences in age, sex ratio, depth of tumor invasion, pTNM classification, or number of positive nodes between the 2 groups. Among patients with positive intrathoracic nodes, the 5-year survival of group B $(\mathbf{4 2} \%)$ was significantly longer than that of group $A(13 \%$, generalized Wilcoxon test $P=$ .02). Among patients with 1 to 4 positive nodes, the 5-year survival of group B (54\%) was significantly higher than that of group A $(22 \%, P=$ $.01)$. Multivariate analysis revealed the number of positive nodes, age, and pT4 stage to be significant predictors of survival in patients with thoracic esophageal carcinoma. Conclusions: Three-field dissection for advanced carcinoma of the thoracic esophagus is effective in patients with 1 to 4 positive nodes. (J Thorac Cardiovasc Surg 1999;117:239-45)
B ecause carcinoma of the thoracic esophagus often involves not only mediastinal lymph nodes but also cervical and abdominal lymph nodes and has a high incidence of locoregional recurrence after radical esophagectomy, long-term survivals are low. For this reason, attempts at en bloc resection of the esophagus have been carried out. ${ }^{1}$ In this decade Japanese surgeons have postulated that 3-field lymphadenectomy (3-field dissection including bilateral cervical, medi-

From the First Department of Surgery, Kumamoto University, School of Medicine, ${ }^{\mathrm{a}}$ Kumamoto, and Izumi City Hospital, ${ }^{\mathrm{b}}$ Izumi City, Kagoshima, Japan.

Received for publication Nov 10, 1997; revisions requested Jan 22, 1998; revisions received July 9, 1998; accepted for publication Sept 16, 1998.

Address for reprints: Yoichi Tabira, MD, First Department of Surgery, Kumamoto University School of Medicine, 1-1-1, Honjo Kumamoto City, Kumamoto 860 -0811, Japan.

Copyright $\odot 1999$ by Mosby, Inc.

$0022-5223 / 99 \$ 8.00+0 \quad \mathbf{1 2} / \mathbf{1} / \mathbf{9 4 6 3 7}$ astinal, and abdominal lymph nodes) improves the survival of patients with carcinoma of the thoracic esophagus. ${ }^{2,3}$ Recently, Altorki and Skinner ${ }^{4}$ reported that cervical node metastases are early events in carcinoma of the esophagus. Although that report suggested the efficacy and necessity of 3-field dissection for the treatment of carcinoma of the esophagus, the benefit of 3field dissection remains controversial. In fact Orringer and Sloan ${ }^{5}$ and Gupta ${ }^{6}$ stressed the superiority of esophagectomy without thoracotomy for carcinoma of the thoracic esophagus because of its low morbidity and mortality rates. They pointed out that severe complications may result from 3-field dissection, especially recurrent nerve palsy caused by the dissection of recurrent nerve nodes. Some surgeons have reported a high incidence $(70 \%)$ of recurrent nerve injury after 3 -field dissection. ${ }^{7}$ This potential morbidity emphasizes the importance of determining which patients are most likely to benefit from 3-field dissection. 
Table I. Characteristics of 86 patients with locally advanced squamous cell carcinoma of the esophagus

\begin{tabular}{lccc}
\hline & $\begin{array}{c}\text { Group A } \\
\text { (2-field dissection, } \\
n=46)\end{array}$ & $\begin{array}{c}\text { Group B } \\
\text { (3-field dissection, } \\
n=40)\end{array}$ & $\mathrm{P}$ \\
\hline Age (y, mean \pm SD) & $64.6 \pm 9.6$ & $61.2 \pm 9.1$ & .10 \\
Gender & & & .97 \\
Male & 37 & 33 & \\
Female & 9 & 7 & .52 \\
Tumor location & & & \\
Upper & 1 & 28 & \\
Middle & 29 & 10 & \\
Lower & 16 & 5 & .12 \\
T category & & 33 & \\
pT2 & 14 & 2 & \\
pT3 & 29 & 8 & \\
pT4 & 3 & 32 & \\
N category & & & \\
pN0 & 14 & 30 & \\
pN1 & 32 & 10 & \\
M category & & & \\
pM0 & & & \\
pM1 & 41 & & \\
No. of positive nodes & $3.4 \pm 4.5$ & & \\
(mean \pm SD) & & & \\
\hline
\end{tabular}

The number of positive nodes has previously been reported to be an important prognostic factor in carcinoma of the esophagus. ${ }^{8}$ This parameter is useful in staging esophageal carcinoma. There has been no study regarding the indication for 3-field dissection in the presence of lymph node metastases, especially with reference to the sites of nodal involvement and the number of positive nodes. The aim of this retrospective study was to evaluate the efficacy of 3-field dissection for advanced carcinoma of the thoracic esophagus and to clarify the indication for this procedure with respect to the presence of lymph node metastasis.

\section{Patients and methods}

From January 1983 to December 1995, 238 patients with squamous cell carcinoma of the thoracic esophagus underwent esophagectomy at Kumamoto University Hospital, Kumamoto, Japan. Among these 238 patients, this retrospective observational study evaluated the 86 who underwent curative esophagectomy with lymph node dissection through a right thoracotomy for tumors classified as pT2 to pT4 in the TNM staging system (International Union Against Cancer). ${ }^{9}$ All of the 86 patients studied received preoperative and postoperative chemotherapy, including cisplatin and 5-fluorouracil. Of the 86 patients, 19 patients had disease categorized as pT2, 62 had disease categorized as pT3, and 5 had disease categorized as pT4. Among the 5 patients with pT4 disease, 2 had tumors invading the pericardium, 2 had tumors invading the thoracic duct, and 1 had a tumor invading lung and pericardium. These 5 patients underwent en bloc esophagectomy including invaded adjacent structures (R0 in the TNM classification). Of the 86 patients, 46 underwent a 2field dissection including mediastinal and abdominal lymph nodes (group A) and 40 underwent a 3-field dissection including bilateral cervical, mediastinal, and abdominal nodes (group B). Seven patients who underwent "pick up" dissection of cervical nodes were included in group A, as reported by Isono and colleagues. ${ }^{2}$ As a principle, 3-field dissection was not performed in patients who were older than 75 years or had any comorbid diseases, such as diabetes mellitus and liver cirrhosis. Four patients in group A had comorbid diseases; 2 had diabetes mellitus without organ failure and 2 had liver cirrhosis. In group B, only 1 patient had mild diabetes mellitus. No patients in this series had any other comorbid diseases.

Subtotal esophagectomy through a right thoracotomy at the fifth intercostal space was performed as described previously. ${ }^{10}$ In brief, periesophageal nodes, bilateral intrathoracic recurrent nerve chain nodes, infracarinal nodes, and infraaortic arch nodes were resected through a right thoracotomy. If 3-field dissection was chosen, bilateral cervical recurrent laryngeal nerve chain nodes, internal jugular nodes, and supraclavicular nodes were dissected in the area from the upper level of thyroid cartilage to the supraclavicular area with bilateral skin collar incisions. After 2- or 3-field dissection, reconstruction was carried out by esophagogastrostomy or esophagocolostomy in the left side of the neck.

Follow-up was complete for all patients until the end of the study or their death. The statuses of all patients were verified by direct contact, mail, or telephone from the patients or their families at least annually. A follow-up duration of 0 was defined as the date of esophagectomy. The duration of postoperative follow-up ranged from 1 to 144 months, with a mean duration of 41.9 months. The mean durations of groups $\mathrm{A}$ and $\mathrm{B}$ were both 41.9 months.

After further grouping patients according to the number of positive nodes, metastases to mediastinal lymph nodes, and metastases to recurrent nerve chain nodes, survival curves for groups A and B were compared. In addition, survivals within group B were compared after stratification according to cervical nodal involvement. In group B the relationship between total number of positive nodes and number of positive noncervical nodes in patients with cervical node metastasis was examined. Potential prognostic factors in predicting longterm survival of these 86 patients were evaluated by means of the Cox proportional hazards regression model. Age, sex ratio, and the number of positive nodes in groups $\mathrm{A}$ and $\mathrm{B}$ were compared with the unpaired Student $t$ test. The relative frequencies of tumor location and $\mathrm{pT}, \mathrm{pN}$, and $\mathrm{pM}$ statuses were compared with the $\chi^{2}$ test. Survival curves and 3- and 5year survival rates were obtained according to the method of Kaplan and Meier. Comparison of survival curves was carried out with the generalized Wilcoxon test. Statistical calculations were conducted with Stat View (Abacus Concepts Inc, Berkeley, Calif). 


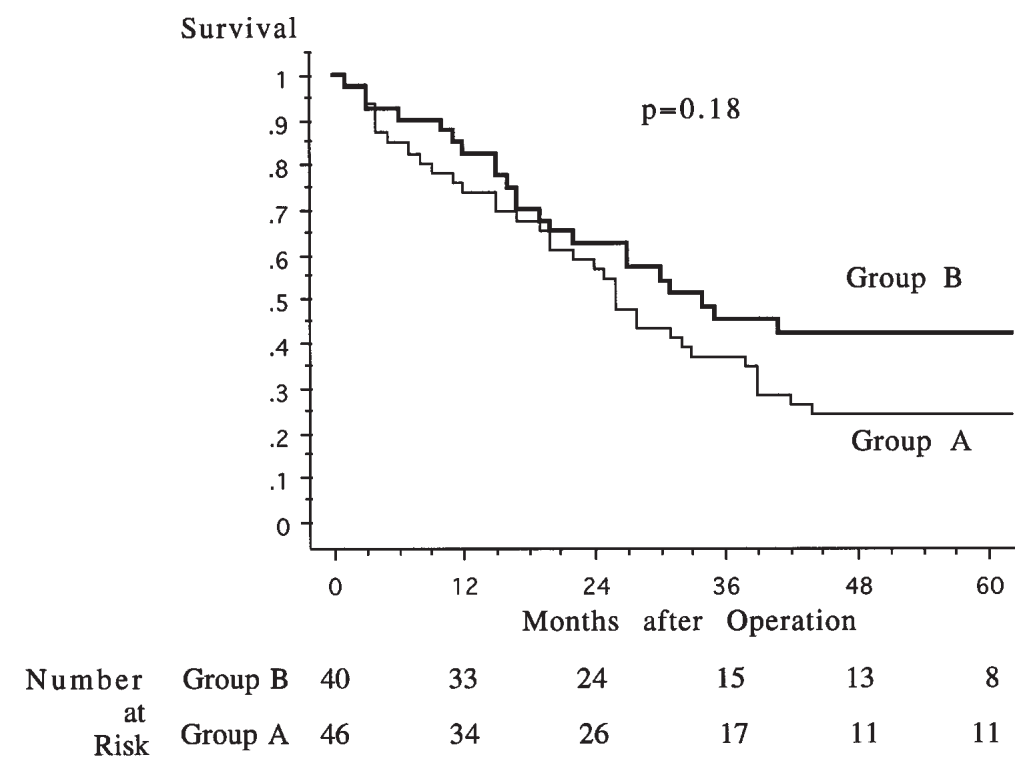

Fig 1. Survival curves of all patients in groups A and B. No significant difference was seen.

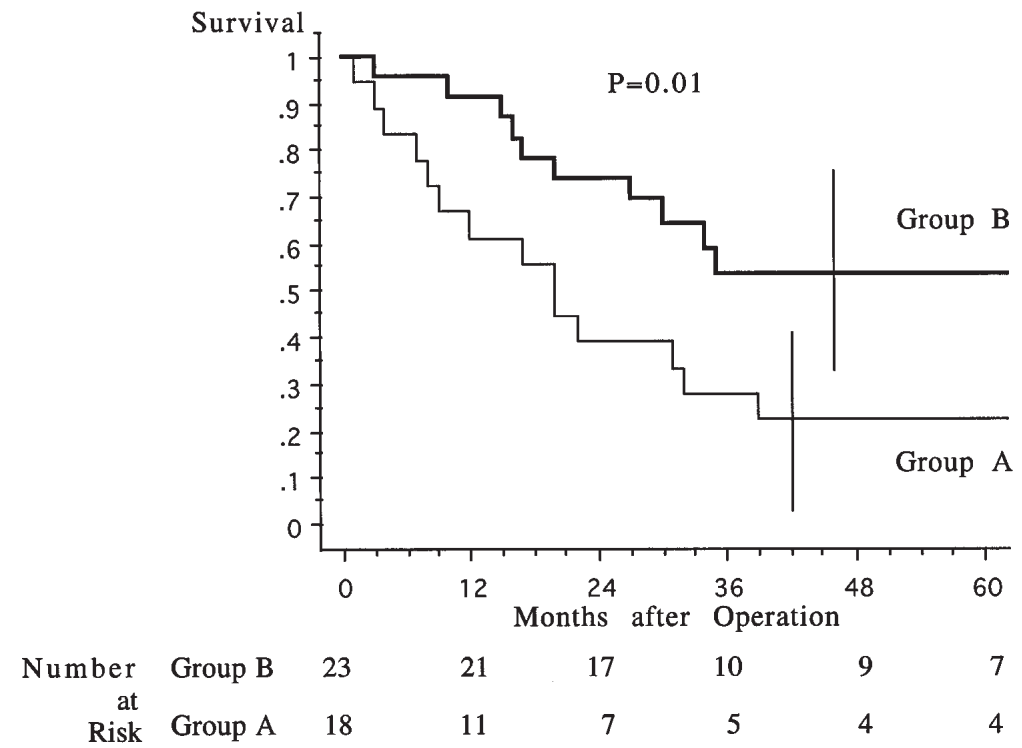

Fig 2. Survival curves of patients with 1 to 4 positive nodes in groups A and B. The vertical bars represent the 95\% confidence interval. A significant difference is seen between the 2 curves.

\section{Results}

Patient characteristics. The patient characteristics of the 2 groups are shown in Table I. The mean ages; the sex ratios (male/female); the distribution of tumor locations; the pT, pN, and pM categories; and the mean number of positive nodes were not significantly different between the 2 groups.

Survivals and number of positive nodes. The 3year and 5-year survivals were $37 \%$ and $24 \%$, respec- tively, for group A and $45 \%$ and $42 \%$, respectively, for group B. No significant difference was seen between the 2 survival curves $(P=.18$; Fig 1$)$. In the subgroup without lymph node metastasis (pN0), the 3-and 5year survivals were $57 \%$ and $43 \%$, respectively, for group A ( $\mathrm{n}=14)$ and both $38 \%$ for group B $(\mathrm{n}=7)$. No significant difference was seen between the 2 survival curves $(P=.48)$. In the subgroup with 1 to 4 positive nodes, the 3 -and 5 -year survivals were $28 \%$ 


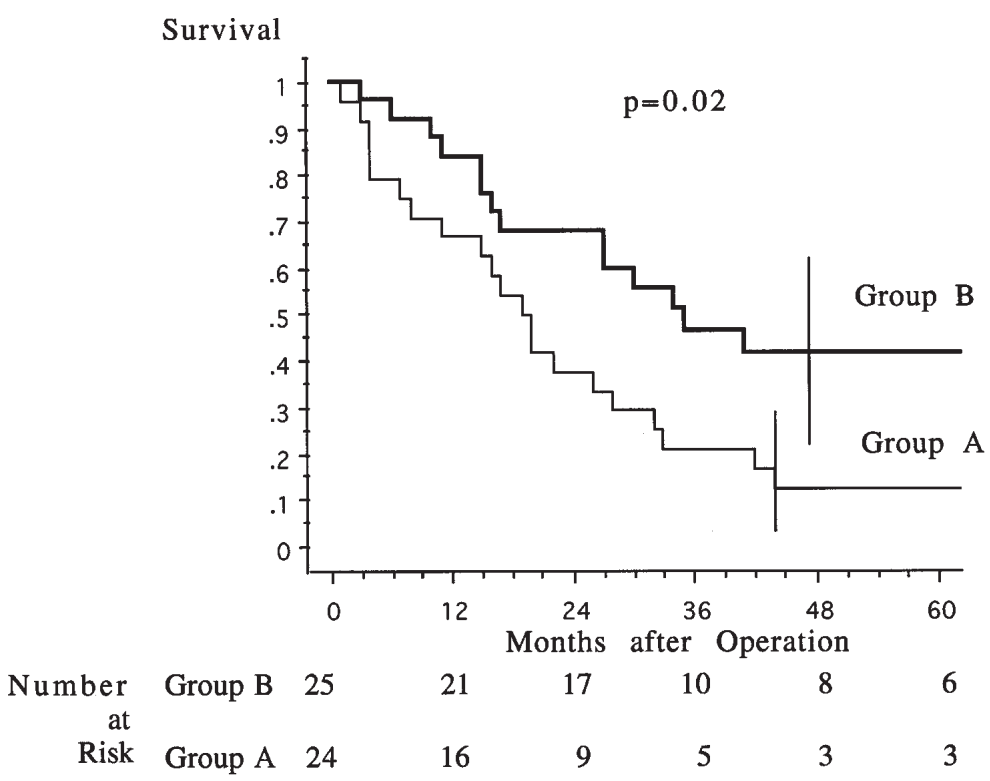

Fig 3. Survival curves of patients with positive intrathoracic nodes in groups A and B. The vertical bars represent the $95 \%$ confidence interval. A significant difference is seen between the 2 curves.

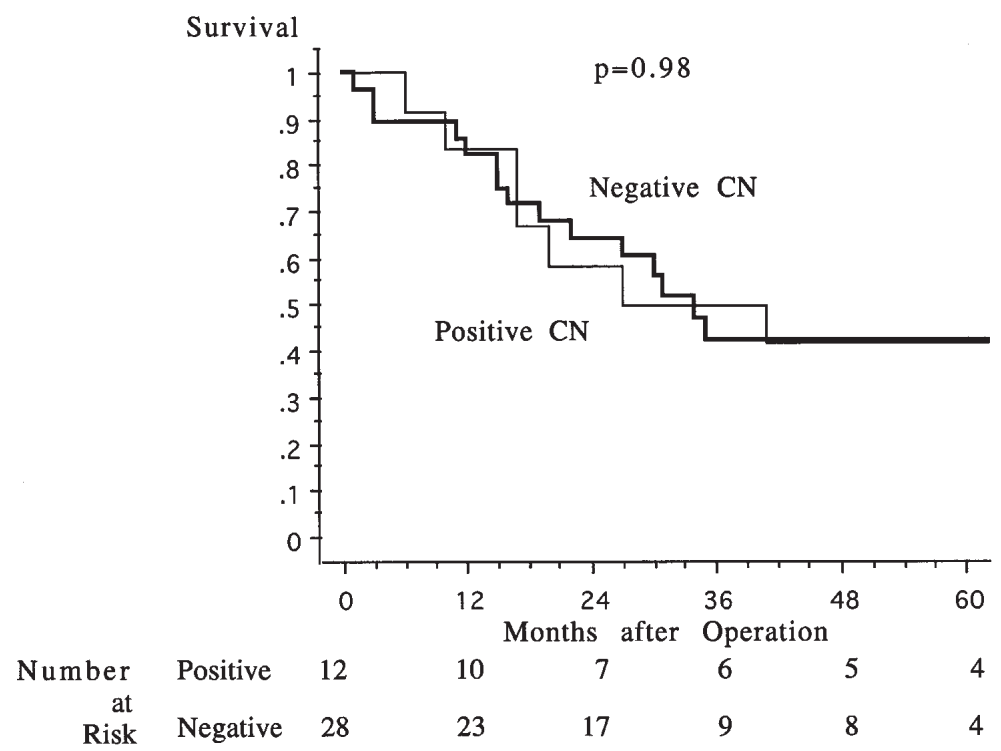

Fig. 4. Survival curves of patients with or without positive cervical nodes who underwent 3 -field dissection. No significant difference was seen. $C N$, Cervical nodes.

and $22 \%$, respectively, for group A $(\mathrm{n}=18)$ and both $54 \%$ for group B $(\mathrm{n}=23)$. Comparison of the 2 survival curves yielded a significant difference $(P=.01$; Fig 2). In the subgroup with 5 or more positive nodes, the 3 - and 5 -year survivals were $21 \%$ and $7 \%$, respectively, for group A $(\mathrm{n}=14)$ and $30 \%$ and $20 \%$, respectively, for group B $(n=10)$. There was no significant difference between the 2 survival curves.

Survivals among patients with positive intrathoracic nodes. Forty-nine of the 86 patients had positive intrathoracic nodes. In this subgroup, the 3- and 5-year survivals were $25 \%$ and $13 \%$, respectively, for group A $(\mathrm{n}=24)$ and $46 \%$ and $42 \%$, respectively, for group B (n $=25)$. There was a significant difference between the 2 survival curves $(P=.02$; Fig 3$)$.

Survivals among patients with positive intrathoracic recurrent nerve chain nodes. Twenty-five patients had positive intrathoracic recurrent nerve chain nodes. Patients with positive pretracheal or right paratracheal nodes also were included in this group. The 3- 
Table II. Relationship of total number of positive nodes and number of positive extracervical nodes in patients with positive cervical nodes who underwent 3-field dissection

\begin{tabular}{lccc}
\hline $\begin{array}{l}\text { Case } \\
\text { No. }\end{array}$ & $\begin{array}{c}\text { Total No. of } \\
\text { positive nodes }\end{array}$ & $\begin{array}{c}\text { No. of } \\
\text { CNs }\end{array}$ & $\begin{array}{c}\text { No. of } \\
\text { ECNs }\end{array}$ \\
\hline 1 & 1 & 1 & 0 \\
2 & 3 & 2 & 1 \\
3 & 3 & 1 & 2 \\
4 & 4 & 2 & 2 \\
5 & 4 & 1 & 3 \\
6 & 4 & 1 & 3 \\
7 & 4 & 1 & 3 \\
8 & 6 & 1 & 5 \\
9 & 9 & 1 & 8 \\
10 & 10 & 1 & 9 \\
11 & 12 & 2 & 10 \\
12 & 31 & 7 & 24 \\
\hline
\end{tabular}

$C N s$, Cervical nodes; $E C N s$, extracervical nodes.

and 5-year survivals were $10 \%$ and $0 \%$, respectively, for group A ( $\mathrm{n}=10)$ and $40 \%$ and $33 \%$, respectively, for group $B(n=15)$. No significant difference was seen between the 2 survival curves $(P=.15)$.

Survivals among patients with positive cervical lymph nodes. In group B, 12 of 40 patients had cervical lymph node metastases. The 3- and 5-year survivals were $50 \%$ and $42 \%$, respectively, for patients with cervical nodal metastases and both $42 \%$ for patients without cervical nodal metastases. No significant difference was seen between the 2 survival curves $(P=.98$; Fig 4$)$.

Relationship between total number of positive nodes and number of positive noncervical nodes in patients with cervical node metastases who underwent 3-field dissection. Table II shows total numbers of positive nodes, numbers of positive cervical nodes, and numbers of positive extracervical nodes (mediastinal and abdominal nodes) in 12 patients in group B with cervical nodal involvement. Of these 12 patients, 7 patients had 1 to 4 positive nodes and 5 patients had 5 or more positive nodes. Of the 7 patients with 1 to 4 positive nodes, 6 patients had 1 to 4 positive nodes in extracervical regions (cases 2-7). Of the 5 patients with 5 or more positive nodes, all had 5 or more positive nodes in extracervical regions (cases 8-12).

Multivariate analysis. The Cox proportional hazards regression model was used to evaluate potential prognostic factors of these 86 patients. Age, sex, comorbidity, tumor location, pT, pN, pM, positive intrathoracic nodes, positive recurrent nerve chain nodes, the number of positive nodes, and 3-field dissection were selected as variables for this analysis. Age, pT4, and the number of positive nodes were
Table III. Results of Cox regression analysis for survival in thoracic esophageal carcinoma

\begin{tabular}{lcll}
\hline Variable & Hazard ratio & $95 \%$ CI & $\mathrm{P}$ \\
\hline Sex (male) & 1.23 & $0.58-2.60$ & .6 \\
Age & 1.04 & $1.01-1.07$ & .02 \\
Comorbid diseases & 0.85 & $0.28-2.61$ & .8 \\
$\begin{array}{l}\text { Tumor location } \\
\quad \text { Upper third }\end{array}$ & 2.07 & $0.38-11.32$ & .4 \\
$\quad$ Middle third & 2.18 & $0.42-11.18$ & .4 \\
T category & & & \\
$\quad$ pT4 & 4.10 & $1.29-13.10$ & .02 \\
$\quad$ pT3 & 0.94 & $0.46-1.93$ & .9 \\
N category & & & \\
$\quad$ pN1 & 0.72 & $0.28-1.85$ & .5 \\
M category & & & \\
$\quad$ pM1 & 0.95 & $0.43-2.10$ & .9 \\
Positive intrathoracic nodes & 1.31 & $0.51-3.36$ & .6 \\
Positive RCNs & 0.95 & $0.45-1.97$ & .9 \\
$\quad$ No. of positive nodes & 1.13 & $1.06-1.20$ & .0002 \\
3-field dissection & 0.57 & $0.31-1.07$ & .08 \\
\hline
\end{tabular}

$C I$, Confidence interval; $R C N S$, recurrent nerve chain nodes.

selected to be significantly predictive of survival after esophagectomy (Table III). The number of positive nodes was thought to be the most strongly predictive because of its $P$ value $(P=.0002)$.

\section{Discussion}

In this study 3-field dissection improved the survival of patients with positive intrathoracic nodes and with 1 to 4 positive nodes but not that of patients without involved nodes (pN0) or with 5 or more positive nodes. The multivariate analysis revealed that the number of positive nodes was a potential prognostic factor. These data suggest that the number of positive nodes could be used as an indication for the performance of a 3-field dissection. The depth of tumor infiltration, as well as lymph node spread, is a potential prognostic factor of esophageal cancer. ${ }^{11}$ Our analysis showed that pT4 cancer was also a significant predictor. Tumors invading to adjacent organs may be beyond the effect of 3 -field dissection, even if they could be resected grossly completely.

Age was also a significant prognostic factor in thoracic esophageal carcinoma. This implies that the indication for 3-field dissection may be regulated by patient's age. In fact, it is reported that high mortality rates have been found among elderly patients undergoing 3-field dissection. ${ }^{12}$

Many surgeons, especially in Japan, have reported that 3-field dissection improves the prognosis associated with carcinoma of the esophagus compared with 2field dissection ${ }^{2,3,13}$; however, no study has addressed the benefit of 3-field dissection with respect to 2-field 
dissection in terms of the number of positive nodes. Ide and coworkers ${ }^{14}$ reported no significant difference in survival between patients with node-negative (pN0) disease who underwent 2- and 3-field dissections. This finding is consistent with our results.

In this study no significant difference between the 2and 3-field groups was seen in the survivals of patients with positive cervical nodes. The mean number of positive nodes in patients with cervical nodal involvement was 6.5 (data not shown). Siewert and colleagues ${ }^{15}$ reported a poor prognosis in patients with 5 or more positive nodes. In a study by Skinner and associates, ${ }^{11}$ penetration though the esophageal wall and lymph node spread were the only factors that influenced survival, and patients with 5 or more positive nodes had a very poor prognosis after en bloc resection. Huang and Sun ${ }^{16}$ reported that the 5-year survival of patients with fewer than 5 positive nodes was significantly higher than that of patients with 5 or more positive nodes. Baba and coworkers ${ }^{12}$ reported that the 5-year survival for patients with 6 or more metastatic nodes was $7.2 \%$, even after 3-field dissection. These results are consistent with our findings and suggest that 3-field dissection is not indicated for patients with 5 or more positive nodes, probably because these patients already have systemic disease. Also, our result showed no difference in survival between the 2 groups with cervical nodal involvement. We reported previously that the survival of patients with positive nodes limited to the recurrent laryngeal nerve chain was better than that of patients with positive internal jugular nodes, including supraclavicular nodes. We concluded that this was because there were more patients with 5 or more positive nodes who had positive internal jugular nodes than who had positive nodes limited to the recurrent laryngeal nerve chain. ${ }^{10}$ These data support our belief that cervical nodal involvement is not a contraindication to 3-field dissection. Recently, identifiable tumor cells in lymph nodes were detected in half of the patients who were considered free of lymph node metastases according to histopathologic analysis after curative en bloc esophagectomy for esophageal cancer. ${ }^{17}$ Our results and those in this report suggest that the addition of cervical node dissection would be beneficial in patients with occult cervical lymph nodes.

Although our multivariate analysis did not find significance in recurrent nerve chain involvement, including pretracheal and right paratracheal nodes, there were no 5-year survivors with positive recurrent nerve chain nodes after 2 -field dissection. Kutsuna ${ }^{18}$ reported that upper mediastinal lymph flow drains mainly into the right cervical lymph nodes. The intrathoracic right recurrent nerve chain nodes were involved in $34.3 \%$ of patients who underwent 3-field dissection, as described previously. ${ }^{19}$ The significance of 3 -field dissection to patients with recurrent nerve chain nodal involvement should be studied further.

It is possible that patients with 1 to 4 positive nodes in group A actually might have had many more positive nodes than were noted, because they did not undergo cervical dissection, and the improved survival seen in this subset was therefore not due to the performance of 3 -field dissection. As mentioned previously, however, no patient with 1 to 4 positive extracervical nodes in group B had 5 or more positive nodes total, and only 1 of the 12 patients with positive cervical nodes in group B (case 1) had the disease "upstaged" as a consequence of the addition of cervical node dissection (Table II). We do not think that there was stage migration in our series.

Three-field dissection for advanced carcinoma of the thoracic esophagus should be limited to patients with 1 to 4 positive nodes. Elderly patients and patients with tumors invading to adjacent structures should especially be excluded from the indication. The clinical strategy derived from these results is as follows. Endoscopic ultrasonography can be used to diagnose depth of tumor infiltrations and lymph node metastases, and its accuracy is $100 \%$ in the preoperative evaluation of esophageal tumors. ${ }^{20}$ Our current criteria of lymph node metastases in preoperative ultrasonography and endoscopic ultrasonography are as follows: (1) hypoechoic pattern and well-demarcated margins, (2) globular shape of lymph node, not flat, and (3) diameter of lymph node larger than $5 \mathrm{~mm}$. The accuracy of this assessment is not perfect, however, because the detective rate of endoscopic ultrasonography is still low in the bilateral recurrent nerve chain nodes. If the preoperatively determined number of positive nodes is 1 to 4 according to these criteria, 3-field dissection is chosen. If preoperative images reveal "bulky" lymph nodes, which implies advanced lymph nodal involvement in many cases, 3-field dissection is not indicated but adjuvant radiochemotherapy is introduced.

\section{REFERENCES}

1. Skinner DB. En bloc resection for neoplasms of the esophagus and cardia. J Thorac Cardiovasc Surg 1983;85:59-71.

2. Isono K, Sato H, Nakayama K. Results of a nationwide study on the three-field lymph node dissection of esophageal cancer. Oncology 1991;48:411-20.

3. Kato H, Watanabe $\mathrm{H}$, Tachimori $\mathrm{Y}$, Iizuka T. Evaluation of neck lymph node dissection for thoracic esophageal carcinoma. Ann Thorac Surg 1991;51:931-5.

4. Altorki NK, Skinner DB. Occult cervical nodal metastasis in 
esophageal cancer: preliminary results of three-field lymphadenectomy. J Thorac Cardiovasc Surg 1997;113:540-4.

5. Orringer MB, Sloan H. Esophagectomy without thoracotomy. J Thorac Cardiovasc Surg 1978;76:634-54.

6. Gupta NM. Oesophagectomy without thoracotomy. Eur J Surg 1996;162:455-61.

7. Fujita H, Kakegawa T, Yamana H, Shima I, Toh Y, Tomita Y, et al. Mortality and morbidity rates, postoperative course, quality of life, and prognosis after extended radical lymphadenectomy for esophageal cancer. Ann Surg 1995;222:654-62.

8. Abe S, Tachibana M, Shiraishi M, Nakamura T. Lymph node metastasis in resectable esophageal cancer. J Thorac Cardiovasc Surg 1990;100:287-91.

9. International Union Against Cancer. TNM classification of malignant tumors. 4th ed, 2nd rev. New York: Springer-Verlag; 1992.

10. Okuma T, Kaneko H, Yoshioka M, Torigoe Y, Miyauchi Y. Prognosis in esophageal carcinoma with cervical lymph node metastasis. Surgery 1993;114:513-8.

11. Skinner DB, Ferguson MK, Soriano A, Little AG, Staszak VM. Selection of operation for esophageal cancer based on staging. Ann Surg 1986;204:391-401.

12. Baba M, Aikou T, Yoshinaka H, Natsugoe S, Fukumoto T, Shimazu H, et al. Long-term results of subtotal esophagectomy with three-field lymphadenectomy for carcinoma of the thoracic esophagus. Ann Surg 1994;219:310-6.

13. Akiyama H, Tsurumaru M, Kajiyama Y. Radical lymph node dis- section for cancer of the thoracic esophagus. Ann Surg 1994; 220:364-73.

14. Ide H, Eguchi R, Nakamura T, Hayashi K, Yoshida K, Hanashi T, et al. Extended lymph node dissection for thoracic esophageal cancer: efficacy of three field dissection based on preoperative staging. Jpn J Gastroenterol Surg 1995;28:951-5.

15. Siewert JR, Holscher AH, Dittler HJ. Preoperative staging and risk analysis in esophageal carcinoma. Hepatogastroenterology 1990;37:382-7.

16. Huang GJ, Sun KL. Prognostic significance of lymph node metastasis in surgical resection of esophageal carcinoma. In: Ferguson MK, Little AG, Skinner DB, editors. Disease of the esophagus, vol 1. Malignant disease. New York: Futura; 1990. p. 213-8.

17. Izbicki JR, Hosch SB, Pichilmeier U, Rehder A, Busch C, Niendorf A, et al. Prognostic value of immunohistochemically identifiable tumor cells in lymph nodes of patients with completely resected esophageal cancer. N Engl J Med 1997;337: 1188-94.

18. Kutsuna M. Intrathoracic lymph flow, with special regard to the communication of intrathoracic lymph vessel with cervical lymph nodes. Kumamoto Med J 1965;18:228-33.

19. Sharma S, Fujita H, Yamana H, Kakegawa T. Patterns of lymph node metastasis in 3-field dissection for carcinoma in the thoracic esophagus. Surg Today Jpn J Surg 1994;24:410-4.

20. Greenberg J, Durkin M, Drunen MV, Aranha GV. Computed tomography or endoscopic ultrasonography in preoperative staging of gastric and esophageal tumors. Surgery 1994;116:696-702.

\section{Authoritative}

The Journal of Thoracic and Cardiovascular Surgery is the most frequently cited thoracic/cardiovascular surgery journal in the Science Citation Index. An article in JTCVS is sited on average almost twice as often as those in the closest cardiothoracic journal. 